\title{
On Some Discrete Boundary Value Problems in Canonical Domains
}

\author{
Alexander V. Vasilyev and Vladimir B. Vasilyev
}

\begin{abstract}
We study some discrete boundary value problems for discrete elliptic pseudo-differential equations in a half-space. These statements are related with a special periodic factorization of an elliptic symbol and a number of boundary conditions depends on an index of periodic factorization. This approach was earlier used by authors for studying special types of discrete convolution equations. Here we consider more general equations and functional spaces.
\end{abstract}

Keywords Disctrete operator - Periodic factorization - Discrete boundary value problem

\section{Introduction}

We will consider a certain class of discrete operators and equations in some so-called canonical domains from Euclidean space $\mathbf{R}^{m}$. These operators are defined by a given function on the $m$-dimensional cube $\mathbf{T}^{m}=[-\pi, \pi]^{m}$, such a function is called a symbol of the discrete operator. Simple examples of such operators have the form

$$
u_{d}(\tilde{x}) \longmapsto \sum_{\tilde{x} \in D_{d}} A_{d}(\tilde{x}-\tilde{y}) u_{d}(\tilde{y}), \quad \tilde{x} \in D_{d},
$$

where $D_{d}=\mathbf{Z}^{m} \cap D, D$ is a domain $D \subset \mathbf{R}^{m}, A_{d}, u_{d}$ are functions of a discrete variable $\tilde{x} \in \mathbf{Z}^{m}$, and the given function $A_{d}(\tilde{x})$ is called a kernel of the operator. Such operators and related ones are called discrete convolutions and were studied from different points of view in a lot of papers (see, for example, [1-9]).

\footnotetext{
A. V. Vasilyev · V. B. Vasilyev (凶)

Belgorod National Research University, Studencheskaya 14/1, Belgorod 308007, Russia e-mail: vladimir.b.vasilyev@gmail.com
}

\author{
A. V. Vasilyev \\ e-mail: alexvassel@gmail.com
}


This paper is devoted to more general operators and equations related to the special canonical domain $D=\mathbf{R}_{+}^{m}=\left\{x \in \mathbf{R}^{m}: x=\left(x_{1}, \ldots, x_{m}\right), x_{m}>0\right\}$ although there are some first results for other canonical domains, for example $D=C_{+}^{a}=\left\{x \in \mathbf{R}^{m}\right.$ : $\left.x=\left(x^{\prime}, x_{m}\right), x_{m}>a\left|x^{\prime}\right|, a>0\right\}[10-12]$.

\section{Discrete Pseudo-differential Operators}

\subsection{Discrete Fourier Transform and Symbols}

Let $u_{d}(\tilde{x})$ be a function of a discrete variable $\tilde{x} \in h \mathbf{Z}^{m}, h>0$. The discrete Fourier transform $F_{d}$ of the function $u_{d}$ is called the following series

$$
\left(F_{d} u_{d}\right)(\xi) \equiv \tilde{u}(\xi) \equiv \sum_{\tilde{x} \in h \mathbf{Z}^{m}} e^{-i \tilde{x} \cdot \xi} u_{d}(\tilde{x}) h^{m}, \quad \xi \in \hbar \mathbf{T}^{m}, \quad \hbar \equiv h^{-1}
$$

if the series converges.

Evidently the function $\tilde{u}(\xi)$ is defined on $\mathbf{R}^{m}$, ant it is a periodic function with basic cube of periods $\hbar \mathbf{T}^{m}$; such functions we call periodic functions.

The Fourier transform is an isomorphism between $L_{2}\left(h \mathbf{Z}^{m}\right)$ and $L_{2}\left(\hbar \mathbf{T}^{m}\right)$, moreover

$$
\left(F_{d}^{-1} \tilde{u}_{d}\right)(\tilde{x})=\frac{1}{(2 \pi)^{m}} \int_{\hbar \mathbf{T}^{m}} e^{i \tilde{x} \cdot \xi} \tilde{u}(\xi) d \xi, \quad \tilde{x} \in h \mathbf{Z}^{m}
$$

Example 1 Let

$$
\left(\Delta_{k}^{(1)} u_{d}\right)(\tilde{x})=\frac{u_{d}\left(\tilde{x}_{1}, \ldots, \tilde{x}_{k}+h, \ldots, \tilde{x}_{m}\right)-u_{d}\left(\tilde{x}_{1}, \ldots, \tilde{x}_{k}, \ldots, \tilde{x}_{m}\right)}{h}
$$

be the divided difference of first order on $\tilde{x}_{k}$, then for its Fourier transform we have

$$
\widetilde{\left(\Delta_{k}^{(1)} u_{d}\right)}(\xi)=\frac{e^{-i \xi_{k}}-1}{h}, \quad \xi \in \mathbf{T}^{m} .
$$

If we consider the divided difference of second order on $\tilde{x}_{k}$

$$
\begin{gathered}
\left(\Delta_{k}^{(2)} u_{d}\right)(\tilde{x})=\hbar^{2}\left(u_{d}\left(\tilde{x}_{1}, \ldots, \tilde{x}_{k}+2, \ldots, \tilde{x}_{m}\right)-2 u_{d}\left(\tilde{x}_{1}, \ldots, \tilde{x}_{k}+1, \ldots, \tilde{x}_{m}\right)+\right. \\
\left.u_{d}\left(\tilde{x}_{1}, \ldots, \tilde{x}_{k}, \ldots, \tilde{x}_{m}\right)\right)
\end{gathered}
$$

then

$$
\left.\widetilde{\left(\Delta_{k}^{(2)} u_{d}\right.}\right)(\xi)=\hbar^{2}\left(e^{-i \xi_{k}}-1\right)^{2} \tilde{u}_{d}(\xi), \quad \xi \in \hbar \mathbf{T}^{m}
$$


Thus, if we introduce so called discrete Laplacian for a function of a discrete variable

$$
\left(\Delta_{d} u_{d}\right)(\tilde{x}) \equiv\left(\sum_{k=1}^{m} \Delta_{k}^{(2)} u_{d}\right)(\tilde{x}), \quad \tilde{x} \in h \mathbf{Z}^{m}
$$

we obtain

$$
\left.\widetilde{\left(\Delta_{d} u_{d}\right.}\right)(\xi)=\hbar^{2} \sum_{k=1}^{m}\left(e^{-i \xi_{k}}-1\right)^{2} \tilde{u}_{d}(\xi), \quad \xi \in \hbar \mathbf{T}^{m}
$$

\subsection{Functional Spaces}

Definition 1 Discrete Sobolev-Slobodetskii space $H^{s}\left(h \mathbf{Z}^{m}\right), s \in \mathbf{R}$, consists of functions for which the following norm

$$
\left\|u_{d}\right\|_{s}=\left(\int_{\hbar \mathbf{T}^{m}}\left(1+\left|\hat{\xi}^{2}\right|\right)^{s}\left|\tilde{u}_{d}(\xi)\right|^{2} d \xi\right)^{1 / 2}
$$

is finite,

$$
\hat{\xi}^{2} \equiv \hbar^{2} \sum_{k=1}^{m}\left(e^{-i h \xi_{k}}-1\right)^{2}
$$

Definition 2 The discrete space $H^{s}\left(h D_{d}\right)$ consists of functions from $H^{s}\left(h \mathbf{Z}^{m}\right)$ for which their supports belong to $\overline{h D_{d}}$. A norm in the space $H^{s}\left(h D_{d}\right)$ is induced by the norm of $H^{s}\left(h \mathbf{Z}^{m}\right)$. The space $H_{+}^{s}\left(h D_{d}\right)$ consists of functions of a discrete variable defined in $h D_{d}$ which admit continuation on the whole $H^{s}\left(h \mathbf{Z}^{m}\right)$. The norm in such a space is given by the formula

$$
\left\|u_{d}\right\|_{s}^{+}=\inf \left\|\ell u_{d}\right\|_{s}
$$

where infimum is taken over all continuations $\ell$.

We will denote by $\widetilde{H}^{s}\left(h D_{d}\right), \widetilde{H}^{s}\left(h \mathbf{Z}^{m} \backslash h D_{d}\right)$ images of the spaces $H^{s}\left(h D_{d}\right)$, $H^{s}\left(h \mathbf{Z}^{m} \backslash h D_{d}\right)$ under discrete Fourier transform $F_{d}$.

Similar functional spaces were introduced and studied in the paper [13], there are a lot of their useful properties. 


\subsection{Periodic Symbols and Discrete Operators}

Definition 3 The function $\widetilde{A}_{d}(\xi) \in C\left(\hbar \mathbf{T}^{m}\right)$ is called a symbol of discrete pseudodifferential operator $A_{d}$, which is defined by the formula

$$
\left(A_{d} u_{d}\right)(\tilde{x})=\frac{1}{(2 \pi)^{m}} \sum_{\tilde{y} \in h \mathbf{Z}^{m}} \int_{\hbar \mathbf{T}^{m}} e^{i \xi \cdot(\tilde{x}-\tilde{y})} \tilde{A}_{d}(\xi) \tilde{u}_{d}(\xi) d \xi, \quad \tilde{x} \in h \mathbf{Z}^{m} .
$$

The symbol $\widetilde{A}_{d}(\xi)$ is called an elliptic symbol if $\widetilde{A}_{d}(\xi) \neq 0, \forall \xi \in \hbar \mathbf{T}^{m}$.

We denoted by $E_{\alpha}$ the class of periodic symbols satisfying the condition

$$
c_{1}\left(1+\left|\hat{\xi}^{2}\right|\right)^{\frac{\alpha}{2}} \leq\left|A_{d}(\xi)\right| \leq c_{2}\left(1+\left|\hat{\xi}^{2}\right|\right)^{\frac{\alpha}{2}}
$$

with constants $c_{1}, c_{2}$ non-depending on $h$.

Remark 1 We use this definition taking into account in future limit transfer from discrete structure to continue one, and

$$
\left|\hat{\xi}^{2}\right| \sim|\xi|^{2}, h \rightarrow 0
$$

Theorem 1 A discrete pseudo-differential operator with symbol $A_{d}(\xi) \in E_{\alpha}$ is a linear bounded operator $A_{d}: H^{s}\left(h \mathbf{Z}^{m}\right) \rightarrow H^{s-\alpha}\left(h \mathbf{Z}^{m}\right)$ with a norm non-depending on $h$.

Each such operator corresponds to the equation

$$
\left(A_{d} u_{d}\right)(\tilde{x})=v_{d}(\tilde{x}), \quad \tilde{x} \in D_{d},
$$

and we will seek the solution $u_{d} \in H^{s}\left(h D_{d}\right)$ for the given right-hand side $v_{d} \in$ $H_{+}^{s}\left(h D_{d}\right)$ and given operator $A_{d}$ with symbol $A_{d}(\xi) \in E_{\alpha}$.

\section{Discrete Equations in a Half-Space}

In this section we study an auxiliary technique for studying solvability of the Eq. (2) for the special case $D=\mathbf{R}_{+}^{m}$.

\subsection{Periodic Hilbert Transform}

We will remind here the classical Hilbert transform and its connections with boundary properties of analytic functions [14-16] and will describe same properties of its periodic analogue. 
The classical Hilbert transform is defined by the following one-dimensional singular integral

$$
(H u)(x)=v \cdot p \cdot \int_{-\infty}^{+\infty} \frac{u(y) d y}{x-y}, \quad x \in \mathbf{R} .
$$

This transform plays key role under studying solvability of model elliptic pseudo-differential equations in a multidimensional half-space $\mathbf{R}_{+}^{m}=\left\{x \in \mathbf{R}^{m}: x=\right.$ $\left.\left(x_{1}, \ldots, x_{m}\right), x_{m}>0\right\}$. Its periodic analogue is the following

$$
\left(H^{p e r} u\right)(x)=\frac{1}{2 \pi i} v \cdot p \cdot \int_{-\pi}^{\pi} \cot \frac{x-y}{2} u(y) d y, \quad x \in[-\pi, \pi] .
$$

It was shown [6] that this periodic singular integral appears under studying discrete equations in the discrete half-space $\mathbf{Z}_{+}^{m}=\mathbf{Z}^{m} \cap \mathbf{R}_{+}^{m}$, also such integrals appear under summation of Fourier series [17].

\subsection{Periodic Riemann Boundary Value Problem}

Let us denote by $P_{+}, P_{-}$projection operators on $h D_{d}, h \mathbf{Z}^{m} \backslash h D_{d}$ respectively. To apply the discrete Fourier transform $F_{d}$ to the Eq. (2) we need to know what are the operators $F_{d} P_{+}, F_{d} P_{-}$. It was done in papers [4, 6], and here we will briefly describe these constructions.

One can define a discrete analogue of the Schwartz space $S\left(h \mathbf{Z}^{m}\right)$ (see for example [13]) and introduce for such functions the following operators which are generated by periodic analogue of the Hilbert transform, $\xi=\left(\xi^{\prime}, \xi_{m}\right)$,

$$
\begin{gathered}
\left(H_{\xi^{\prime}}^{p e r} \tilde{u}_{d}\right)(\xi)=\frac{1}{2 \pi i} v \cdot p \cdot \int_{-\hbar \pi}^{\hbar \pi} \cot \frac{\hbar\left(\xi_{m}-\eta_{m}\right)}{2} \tilde{u}_{d}\left(\xi^{\prime}, \eta_{m}\right) d \eta_{m}, \quad \xi^{\prime} \in \hbar \mathbf{T}^{m-1}, \\
P_{\xi^{\prime}}^{\text {per }}=1 / 2\left(I+H_{\xi^{\prime}}^{\text {per }}\right), \quad Q_{\xi^{\prime}}^{\text {per }}=1 / 2\left(I-H_{\xi^{\prime}}^{\text {per }}\right) .
\end{gathered}
$$

Lemma 1 We have the following relations

$$
F_{d} P_{+}=P_{\xi^{\prime}}^{\text {per }} F, \quad F_{d} P_{-}=Q_{\xi^{\prime}}^{\text {per }} F
$$

The Lemma 1 implies that a solvability of the Eq. (2) is closely related to a solvability of one-dimensional singular integral equation with the periodic Hilbert transform and a parameter $\xi^{\prime} \in \hbar \mathbf{T}^{m-1}$. The last equation can be solved with a help of so called 
periodic Riemann problem [6] which is formulated as followings. Let us denote by $\Pi_{ \pm}$the upper and lower half-strips in a complex plane $\mathbf{C}$,

$$
\Pi_{ \pm}=\{z \in \mathbf{C}: z=t+i s, t \in[-\pi, \pi], \pm s>0\} .
$$

The problem is the following. Finding two functions $\Phi^{ \pm}(t), t \in[-\pi, \pi]$ (from appropriate functional spaces), which admit an analytical continuation into $\Pi_{ \pm}$and satisfy the linear relation

$$
\Phi^{+}(t)=G(t) \Phi^{-}(t)+g(t)
$$

where $G(t), g(t)$ are given functions on $[-\pi, \pi], G(-\pi)=G(\pi), g(-\pi)=g(\pi)$. If $G(t) \equiv 1$ then the problem (3) is called a jump problem.

Lemma 2 For $|s|<1 / 2$, the operators $P_{\xi^{\prime}}^{\text {per }}, Q_{\xi^{\prime}}^{\text {per }}$ are bounded projectors $P_{\xi^{\prime}}^{\text {per }}$ : $\widetilde{H}^{s}\left(h \mathbf{Z}^{m}\right) \rightarrow \widetilde{H}^{s}\left(h D_{d}\right), Q_{\xi^{\prime}}^{\text {per }}: \widetilde{H}^{s}\left(h \mathbf{Z}^{m}\right) \rightarrow \widetilde{H}^{s}\left(h \mathbf{Z}^{m} \backslash h D_{d}\right)$, and a jump problem has unique solution $\Phi^{+} \in \widetilde{H}^{s}\left(h D_{d}\right), \Phi^{-} \in \widetilde{H}^{s}\left(h \mathbf{Z}^{m} \backslash h D_{d}\right)$ for arbitrary $g \in$ $\widetilde{H}^{s}\left(h \mathbf{Z}^{m}\right)$,

$$
\Phi^{+}=P_{\xi^{\prime}}^{\text {per }} g, \quad \Phi^{-}=-Q_{\xi^{\prime}}^{\text {per }} g
$$

\subsection{Periodic Factorization}

To study the general Riemann boundary value problem (3) we will use the following concept.

Definition 4 Periodic factorization of an elliptic symbol $A_{d}(\xi) \in E_{\alpha}$ is called its representation in the form

$$
A_{d}(\xi)=A_{d,+}(\xi) A_{d,-}(\xi)
$$

where the factors $A_{d, \pm}(\xi)$ admit an analytical continuation into half-strips $\hbar \Pi_{ \pm}$on the last variable $\xi_{m}$ for all fixed $\xi^{\prime} \in \hbar \mathbf{T}^{m-1}$ and satisfy the estimates

$$
\left|A_{d,+}^{ \pm 1}(\xi)\right| \leq c_{1}\left(1+\left|\hat{\zeta}^{2}\right|\right)^{ \pm \frac{x}{2}}, \quad\left|A_{d,-}^{ \pm 1}(\xi)\right| \leq c_{2}\left(1+\left|\hat{\zeta}^{2}\right|\right)^{ \pm \frac{\alpha-x}{2}}
$$

with constants $c_{1}, c_{2}$ non-depending on $h$,

$$
\hat{\zeta}^{2} \equiv \hbar^{2}\left(\sum_{k=1}^{m-1}\left(e^{-i h \xi_{k}}-1\right)^{2}+\left(e^{-i h\left(\xi_{m}+i \tau\right)}-1\right)^{2}\right), \quad \xi_{m}+i \tau \in \hbar \Pi_{ \pm} .
$$

The number $æ \in \mathbf{R}$ is called an index of periodic factorization. 
For some simple cases one can use the topological formula

$$
æ=\frac{1}{2 \pi} \int_{-\hbar \pi}^{\hbar \pi} d \arg A_{d}\left(\cdot, \xi_{m}\right),
$$

where $A_{d}\left(\cdot, \xi_{m}\right)$ means that $\xi^{\prime} \in \hbar \mathbf{T}^{m-1}$ is fixed, and the integral is the integral in Stieltjes sense. It means that we need to calculate divided by $2 \pi$ variation of the argument of the symbol $A_{d}(\xi)$ when $\xi_{m}$ varies from $-\hbar \pi$ to $\hbar \pi$ under fixed $\xi^{\prime}$.

Example 2 Let $A_{d}(\xi)=k^{2}+\hat{\xi}^{2}, k \in \mathbf{R}$, such that the condition (1) is satisfied, in other words $A_{d}$ is the discrete Laplacian plus $k^{2} I$. The variation of an argument mentioned above can be calculated immediately, and it equals to 1 .

\section{Solvability}

As we will see the index of factorization very influences on the solvability picture of the Eq. (3).

\subsection{Existence and Uniqueness Theorem}

Theorem 2 If the elliptic symbol $\tilde{A}_{d}(\xi) \in E_{\alpha}$ admits periodic factorization with index æ so that $|æ-s|<1 / 2$ then the Eq.(2) has unique solution in the space $H^{s}\left(h D_{d}\right)$ for arbitrary right-hand side $v_{d} \in H^{s-\alpha}\left(h D_{d}\right)$.

Proof Let $\ell v_{d}$ be an arbitrary continuation of $v_{d}$ on the whole $h \mathbf{Z}^{m}$ so that $\ell v_{d} \in$ $H^{s-\alpha}\left(h \mathbf{Z}^{m}\right)$. Let

$$
w_{d}(\tilde{x})=\left(\ell v_{d}\right)(\tilde{x})-\left(A_{d} u_{d}\right)(\tilde{x})
$$

and rewrite

$$
\left(A_{d} u_{d}\right)(\tilde{x})+w_{d}(\tilde{x})=\left(\ell v_{d}\right)(\tilde{x}) .
$$

Further applying the discrete Fourier transform $F_{d}$ and using the periodic factorization we write

$$
\tilde{A}_{d,+}(\xi) \tilde{u}_{d}(\xi)+\tilde{A}_{d,-}^{-1}(\xi) \tilde{w}_{d}(\xi)=\tilde{A}_{d,-}^{-1}(\xi) \widetilde{\ell v_{d}}(\xi)
$$

According to Theorem 1 we have $\tilde{A}_{d,+}(\xi) \tilde{u}_{d}(\xi) \in \widetilde{H}^{s-\mathfrak{x}}\left(h \mathbf{Z}^{m}\right), \tilde{A}_{d,-}^{-1}(\xi) \tilde{w}_{d}(\xi) \in$ $\widetilde{H}^{s-\alpha+\alpha-\mathfrak{x}}\left(h \mathbf{Z}^{m}\right)$ and analogously $\tilde{A}_{d,-}^{-1}(\xi) \widetilde{\ell v_{d}}(\xi) \in \widetilde{H}^{s-\mathfrak{x}}\left(h \mathbf{Z}^{m}\right)$. Moreover, really $\tilde{A}_{d,+}(\xi) \tilde{u}_{d}(\xi) \in \tilde{H}^{s-\mathfrak{x}}\left(h D_{d}\right)$ in view of a holomorphy property, and accurate con- 
siderations with supports of $A_{d,-}(\xi)$ and $\tilde{w}_{d}(\xi)$ show that in fact $\tilde{A}_{d,-}^{-1}(\xi) \tilde{w}_{d}(\xi) \in$ $\widetilde{H}^{s-\mathfrak{x}}\left(h \mathbf{Z}^{m} \backslash h D_{d}\right)$.

Thus we obtain a variant of a jump problem for the space $\widetilde{H}^{s-\mathfrak{x}}\left(h \mathbf{Z}^{m}\right)$ which can be solved by the Lemma2. According to this lemma we have

$$
\tilde{A}_{d,+}(\xi) \tilde{u}_{d}(\xi)=P_{\xi^{\prime}}^{p e r}\left(\tilde{A}_{d,-}^{-1}(\xi) \widetilde{\ell v_{d}}(\xi)\right)
$$

or finally

$$
\tilde{u}_{d}(\xi)=\tilde{A}_{d,+}^{-1}(\xi) P_{\xi^{\prime}}^{p e r}\left(\tilde{A}_{d,-}^{-1}(\xi) \widetilde{\ell v_{d}}(\xi)\right)
$$

It finishes the proof.

Remark 2 It is easy to see that the solution does not depend on choice of continuation $\ell v_{d}$.

\subsection{A General Solution of the Discrete Equation}

Here we consider more complicated case when the condition $|æ-s|<1 / 2$ does not hold. There are two possibilities in this situation, and we consider one case which leads to typical boundary value problems.

Theorem 3 Let $æ-s=n+\delta, n \in \mathbf{N},|\delta|<1 / 2$. Then a general solution of the Eq.(2) in Fourier images has the following form

$$
\tilde{u}_{d}(\xi)=\tilde{A}_{d,+}^{-1}(\xi) X_{n}(\xi) P_{\xi^{\prime}}^{p e r}\left(X_{n}^{-1}(\xi) \tilde{A}_{d,-}^{-1}(\xi) \widetilde{\ell v_{d}}(\xi)\right)+\tilde{A}_{d,+}^{-1}(\xi) \sum_{k=0}^{n-1} c_{k}\left(\xi^{\prime}\right) \hat{\zeta}_{m}^{k}
$$

where $X_{n}(\xi)$ is an arbitrary polynomial of order $n$ of variables $\hat{\zeta}_{k}=\hbar\left(e^{-i h \xi_{k}}-\right.$ $1), k=1, \ldots, m$, satisfying the condition (1), $c_{k}\left(\xi^{\prime}\right), j=0,1, \ldots, n-1$, are arbitrary functions from $H_{s_{k}}\left(h \mathbf{T}^{m-1}\right), s_{k}=s-\mathfrak{Q}+k-1 / 2$.

The Theorem 3 implies that if we want to have a unique solution in the case $æ-s=n+\delta, n \in \mathbf{N},|\delta|<1 / 2$, we need some additional conditions to determine uniquely unknown functions $c_{k}\left(\xi^{\prime}\right), k=0,1, \ldots, n-1$. This case we will discuss in the next section.

Corollary 1 Let $æ-s=n+\delta, \in \mathbf{N},|\delta|<1 / 2, v_{d} \equiv 0$. A general solution of the equation (2) has the following form

$$
\tilde{u}_{d}\left(\tilde{x}^{\prime}, \tilde{x}_{m}\right)=\tilde{A}_{d,+}^{-1}(\xi) \sum_{k=0}^{n-1} c_{k}\left(\xi^{\prime}\right) \hat{\zeta}_{m}^{k}
$$




\section{Boundary Value Problems}

This section is a direct continuation of the previous one and gives a statement of simple boundary value problem for the Eq. (2). We start from a formula for general solution for the Eq. (2) including unknown functions $c_{k}\left(\xi^{\prime}\right), k=0,1 \ldots, n-1$. For simplicity we consider a homogeneous equation (2) although all results will be valid for inhomogeneous case without additional special requirements.

Let us introduce the following boundary conditions

$$
\left(B_{j} u_{d}\right)\left(\tilde{x}^{\prime}, 0\right)=b_{j}\left(\tilde{x}^{\prime}\right), \quad j=0,1 \ldots, n-1,
$$

$\underset{\widetilde{B}}{\text { where }} B_{d, j}$ be a discrete pseudo-differential operators of order $\alpha_{j} \in \mathbf{R}$ with symbols $\widetilde{B}_{j}(\xi) \in C\left(\hbar \mathbf{T}^{m}\right)$

$$
\left(B_{d, j} u_{d}\right)(\tilde{x})=\frac{1}{(2 \pi)^{m}} \int_{\hbar \mathbf{T}^{m}} \sum_{\tilde{y} \in h \mathbf{Z}^{m}} e^{i \xi \cdot(\tilde{x}-\tilde{y})} \widetilde{B}_{j}(\xi) \tilde{u}_{d}(\xi) d \xi .
$$

One can rewrite boundary conditions (4) in Fourier images

$$
\int_{-h^{-1} \pi}^{h^{-1} \pi} \widetilde{B}_{j}\left(\xi^{\prime}, \xi_{m}\right) \tilde{u}_{d}\left(\xi^{\prime}, \xi_{m}\right) d \xi_{m}=\tilde{b}_{j}\left(\xi^{\prime}\right), \quad j=0,1 \ldots, n-1,
$$

so that according to properties of pseudo-differential operators (Theorem 1 ) and trace properties [13] we need to require $b_{j}\left(\tilde{x}^{\prime}\right) \in H^{s-\alpha_{j}-1 / 2}\left(h \mathbf{Z}^{m-1}\right)$.

Let us denote

$$
s_{j k}\left(\xi^{\prime}\right)=\int_{-\hbar \pi}^{\hbar \pi} \tilde{A}_{d,+}^{-1}(\xi) \widetilde{B}_{j}\left(\xi^{\prime}, \xi_{m}\right) \hat{\zeta}_{m}^{k} d \xi_{m}
$$

Now we can formulate the following result.

Theorem 4 If $\mathfrak{x}-s=n+\delta, n \in \mathbf{N},|\delta|<1 / 2$, then the boundary value problem (2) and (4) has a unique solution in the space $H^{s}\left(h D_{d}\right)$ for arbitrary $b_{j} \in H^{s-\alpha_{j}-1 / 2}\left(h \mathbf{Z}^{m-1}\right), j=0, \ldots, n-1$, iff

$$
\operatorname{det}\left(s_{k j}\left(\xi^{\prime}\right)\right)_{k, j=0}^{\mathfrak{x}} \neq 0, \quad \forall \xi^{\prime} \in \mathbf{T}^{m-1} .
$$

A priori estimate holds

$$
\left\|u_{d}\right\|_{s} \leq c \sum_{j=0}^{n-1}\left[b_{j}\right]_{s-\alpha_{j}-1 / 2}
$$

where $c$ does not depend on $h$, and $[\cdot]_{s}$ denotes $H^{s}$-norm in the space $H^{s}\left(h \mathbf{Z}^{m-1}\right)$. 
Proof Substituting the general solution of the Eq. (2) into boundary conditions (5) we have

$$
\int_{-\hbar \pi}^{\hbar \pi} \tilde{A}_{d,+}^{-1}(\xi) \widetilde{B}_{j}\left(\xi^{\prime}, \xi_{m}\right) \sum_{k=0}^{n-1} c_{k}\left(\xi^{\prime}\right) \hat{\zeta}_{m}^{k} d \xi_{m}=\tilde{b}_{j}\left(\xi^{\prime}\right), \quad j=0,1 \ldots, n-1,
$$

and further

$$
\sum_{k=0}^{n-1} c_{k}\left(\xi^{\prime}\right) \int_{-\hbar \pi}^{\hbar \pi} \tilde{A}_{d,+}^{-1}(\xi) \widetilde{B}_{j}\left(\xi^{\prime}, \xi_{m}\right) \hat{\zeta}_{m}^{k} d \xi_{m}=\tilde{b}_{j}\left(\xi^{\prime}\right), \quad j=0,1 \ldots, n-1,
$$

Thus, we obtain the following system of linear algebraic equations

$$
\sum_{k=0}^{n-1} s_{j k}\left(\xi^{\prime}\right) c_{k}\left(\xi^{\prime}\right)=\tilde{b}_{j}\left(\xi^{\prime}\right), \quad j=0,1 \ldots, n-1,
$$

with respect to unknown functions $c_{k}\left(\xi^{\prime}\right), k=0,1 \ldots, n-1$. The condition (6) is necessary and sufficient for a unique solvability of inhomogeneous system.

A priori estimates can be easily obtained using properties of pseudo-differential operators and appropriate properties of discrete $H^{S}$-spaces.

The condition (6) is a variant of Shapiro-Lopatinskii condition [18].

Acknowledgements This work was supported by the State contract of the Russian Ministry of Education and Science (contract No 1.7311.2017/B).

\section{References}

1. Gohberg, I.C., Feldman, I.A.: Convolution Equations and Projection Methods for Their Solution. AMS, Providence (1974)

2. Kozak, A.V., Simonenko, I.B.: Projection methods for the solution of multidimensional discrete equations in convolutions. Sib. Math. J. 21, 235-242 (1980)

3. Rabinovich, V.: Wiener algebra of operators on the lattice $\left(\mu Z^{n}\right)$ depending on the small parameter $\mu>0$. Complex Var. Elliptic Equ. 58, 751-766 (2013)

4. Vasilyev, A.V., Vasilyev, V.B.: Discrete singular operators and equations in a half-space. Azerb. J. Math. 3, 84-93 (2013)

5. Vasilyev, A.V., Vasilyev, V.B.: Discrete singular integrals in a half-space. In: Mityushev, V., Ruzhansky, M. (eds.) Current Trends in Analysis and Its Applications. Proceedings of the 9th ISAAC Congress, Krakôw 2013, pp. 663-670. Birkhäuser, Basel (2015)

6. Vasil'ev, A.V., Vasil'ev, V.B.: Periodic Riemann problem and discrete convolution equations. Differ. Equ. 51, 652-660 (2015)

7. Vasilyev, A.V., Vasilyev, V.B.: Difference equations and boundary value problems. In: Pinelas, S., Dŏslá, Z., Dŏslý, O., Kloeden, P. (eds.) Differential and Difference Equations and Applications. Springer Proceedings in Mathematics \& Statistics, vol. 164, pp. 132-421 (2016) 
8. Vasilyev, A.V., Vasilyev, V.B.: On solvability of some difference-discrete equations. Opusc. Math. 36, 525-539 (2016)

9. Vasilyev, A.V., Vasilyev, V.B.: Difference equations in a multidimensional space. Math. Model. Anal. 21, 336-349 (2016)

10. Vasil'ev, V.B.: Wave Factorization of Elliptic Symbols: Theory and Applications. Introduction to the Theory of Boundary Value Problems in Non-smooth Domains. Kluwer Academic Publishers, Dordrecht (2000)

11. Vasilyev, V.: Discrete equations and periodic wave factorization. AIP Conf. Proc. 1759, 020126 (2016). https://doi.org/10.1063/1.4959740

12. Vasilyev, V.: The periodic Cauchy kernel, the periodic Bochner kernel, discrete pseudodifferential operators. AIP Conf. Proc. 1863, 140014 (2017). https://doi.org/10.1063/1. 4992321

13. Frank, L.S.: Spaces of network functions. Math. USSR Sb. 15, 183-226 (1971)

14. Gakhov, F.D.: Boundary Value Problems. Dover Publications, New York (1981)

15. Muskhelishvili, N.I.: Singular Integral Equations. North Holland, Amsterdam (1976)

16. King, F.W.: Hilbert Transforms, vol. 1-2. Cambridge University Press, Cambridge (2009)

17. Edwards, R.E.: Fourier Series. A Modern Introduction, vol. 1-2. Springer, Berlin (1979)

18. Eskin, G.: Boundary Value Problems for Elliptic Pseudodifferential Equations. AMS, Providence (1981) 